\title{
In silico Drug Repurposing for COVID-19: Targeting SARS-CoV-2 Proteins through Docking and Quantum Mechanical Scoring
}

\author{
Claudio N. Cavasotto ${ }^{*,+,}, \boldsymbol{\Phi}$ and Juan I. Di Filippo \\ $\dagger$ Computational Drug Design and Biomedical Informatics Laboratory, Translational \\ Medicine Research Institute (IIMT), CONICET-Universidad Austral, Pilar, Buenos Aires, \\ Argentina \\ $\ddagger$ Facultad de Ciencias Biomédicas, and Facultad de Ingeniería, Universidad Austral, Pilar, \\ Buenos Aires, Argentina \\ \Austral Institute for Applied Artificial Intelligence, Universidad Austral, Pilar, Buenos \\ Aires, Argentina \\ E-mail: CCavasotto@austral.edu.ar
}

\begin{abstract}
In December 2019, an infectious disease caused by the coronavirus SARS-CoV-2 appeared in Wuhan, China. This disease (COVID-19) spread rapidly worldwide, and on March 2020 was declared a pandemic by the World Health Organization (WHO). Today, almost 1,5 million people have been infected, with more than 85,000 casualties. Today, no vaccine nor antiviral drug is available. While the development of a vaccine might take at least a year, and for a novel drug, even longer; finding a new use to an old drug (drug repurposing) could be the most effective strategy. We present a dockingbased screening using a quantum mechanical scoring of a library built from approved
\end{abstract}


drugs and compounds undergoing clinical trials, against three SARS-CoV-2 target proteins: the spike or S-protein, and two proteases, the main protease and the papain-like protease. The S-protein binds directly to the Angiotensin Converting Enzyme 2 receptor of the human host cell surface, while the two proteases process viral polyproteins. Following the anaylysis of our structure-based compound screening, we propose several structurally diverse compounds (either FDA-approved or in clinical trials) that could display antiviral activity against SARS-CoV-2. Clearly, these compounds should be further evaluated in experimental assays and clinical trials to confirm their actual activity against the disease. We hope that these findings may contribute to the rational drug design against COVID-19.

\section{Introduction}

Coronaviruses (CoVs) usually are the cause of mild to serious respiratory tract infections. In the past decades, two highly pathogenic CoVs, the severe acute respiratory syndrome coronavirus (SARS-CoV) and the Middle East respiratory syndrome coronavirus (MERS$\mathrm{CoV}$ ), both transmitted from animals to humans, triggered global epidemics, in 2003 and 2012, respectively, with high mortality rates. ${ }^{1}$ In December 2019, a coronavirus infectious disease (named COVID-19) was detected in Wuhan, province of Hubei, China, caused by a new pathogenic $\mathrm{CoV}$, named SARS-CoV-2. The virus spread very rapidly from China to all countries, and on March 11th, 2020, it was declared a pandemic by the World Health Organization (WHO). At the time of this writing, there are almost 1,5 million cases worldwide, with more than 85,000 fatalities ${ }^{2}$ no country has been spared of this disease. The mortality rate of the SARS-CoV-2 is currently estimated in the range of 0.5-6\%; and while COVID-19

appears to be less deadly than SARS $(\sim 10 \%)^{3}$ or MERS $\left.(\sim 40 \%)\right)^{3}$ it seems to be more contagious, with a reproductive number $\left(R_{o}\right)$ in the range 2.0-6.5, ${ }^{4}$ higher than SARS and MERS, which could explain the velocity of its propagation.

Today, no specific therapeutics are available, and current disease management is limited 
to social measures, such as social distancing, travel ban, and full lockdown in many cities. Thus, there is an urgent need for the discovery of prevention and treatment strategies for the COVID-19. It is acknowledged that the development and evaluation of a vaccine might take at least a year. While the discovery and evaluation of a new drug should take even longer, the use of an existing drug (or compound undergoing clinical trials) to treat COVID-19 (drug repurposing) seems the fastest strategy, since these compounds have either regulatory approval as drugs or have cleared safety studies that indicate a therapeutic potential.

While some pharmaceuticals are currently being tested, ${ }^{5,6}$ there is a clear need to develop new alternatives within the drug repurposing approach. In this contribution, we perform molecular docking-based virtual screening using quantum mechanical (QM) scoring, ${ }^{7-9}$ and a docking library built from approved Food and Drug Administration (FDA) drugs and compounds undergoing clinical trial on the following three viral target proteins: the spike glycoprotein (S-protein), and two proteases, namely, the 3-chymotrypsin-like protease $\left(3 \mathrm{CL}^{\mathrm{pro}}\right)$ [or main protease $\left.\left(\mathrm{M}^{\mathrm{pro}}\right)\right]$, and the papain-like protease $\left(\mathrm{PL}^{\mathrm{pro}}\right)$. The S-protein binds directly to the Angiotensin Converting Enzyme 2 (ACE2) receptor of the human host cell surface -thus enabling virus entry and replication, and the two proteases process the two viral polyproteins encoded by the open reading frames (ORFs) orf1a and orf1b. It has been shown that the PL $\mathrm{Pro}^{\text {pro }}$ also have de-ubiquitination and de-ISGylation activities in SARS and MERS.

From our virtual screening, we suggest a variety of compounds (either FDA-approved or in clinical trials) that may inhibit SARS-CoV-2. Needless to say, these compounds should be evaluated in ad hoc experimental assays and clinical trials to confirm their actual activity against COVID-19. This contribution complements earlier works in the field, ${ }^{10-17}$ and it is our hope to thus collaborate to the worldwide efforts to deliver a prompt answer to the deadly threat of COVID-19. 


\section{Results and Discussion}

We docked a chemical library of 11,552 compounds (composed by FDA-approved, investigational and experimental drugs, cf. the Methods section for details) onto three different proteins of the SARS-CoV-2: the M $\mathrm{M}^{\text {pro }}$, the PL $\mathrm{P}^{\text {pro }}$, and the S-protein. In all targets, molecules were scored with the QM docking score (QMDS). ${ }^{7} \mathrm{QM}$ methods capture the underlying physics of the molecular system accounting for all energy contributions, including electronic polarization, covalent-bond formation, and charge transfer. ${ }^{8}$ For each target, we then analyzed the $1 \%$ top-scoring molecule subset, selecting candidates in terms of their QMDS, the presence of key interactions with the receptor, and visual inspection.

For the $\mathrm{M}^{\text {pro }}$, we used two experimental structures: $\mathrm{M}^{\text {pro }}$ bound to peptide N3 (PDB $6 \mathrm{LU} 7)^{18}$ and the unbound form of the protein (PDB 6YB7). The purpose of using two structures for docking is to account to some extent for protein flexibility by receptor ensemble docking. ${ }^{19,20}$ After docking, scoring, and ranking the molecules of the chemical library on each receptor, the hit-lists were merged and the best ranking pose for each molecule was kept (according to the merging-and-shrinking method ${ }^{21,22}$ ).

Self-docking of peptide N3 onto structure 6LU7 had a root-medium-square deviation (RMSD) of $1.7 \AA$ (an excellent result for a peptide), while according to its QMDS, N3 was within the top $0.3 \%$ of the docked library; this is a simple but necessary validation of our approach. Following the criteria described above, we hereby describe a list of potential inhibitors: Ritonavir (DrugBank ID DB00503) (Figure 1), Indinavir (DB00224) and Lopinavir (DB01601), which are FDA-approved drugs which act as HIV protease inhibitors, and are used to treat HIV/AIDS; Brilacidin (ChEMBL ID CHEMBL2219413) (Figure 1) an investigational drug for the supportive care of mucositis, stomatitis, and head and neck neoplasms (interestingly, Brilacidin is being investigated for direct inhibition of SARS-CoV-2 (press release of April 6th, 2010 23); Samatasvir (CHEMBL3039519) an investigational drug that has been used in trials as a treatment for hepatitis C infection; CR665 (DB05155) an investigational drug for the development of highly selective peripheral $\kappa$-opioid receptor agonists. 
Considering the large size of the binding site, other larger molecules have been identified, such as Antiogensinamide (DB13517), a potent vasoconstrictor used as a cardiac stimulant, and Felypressin (DB00093), an investigational synthetic peptide used as a vasoconstrictor. Other experimental drugs have been also identified, such as DB02747, DB04692, DB04722, and DB03311 (Figure 2). A list of potential inhibitors of the $\mathrm{M}^{\text {pro }}$ is summarized in Table 1.

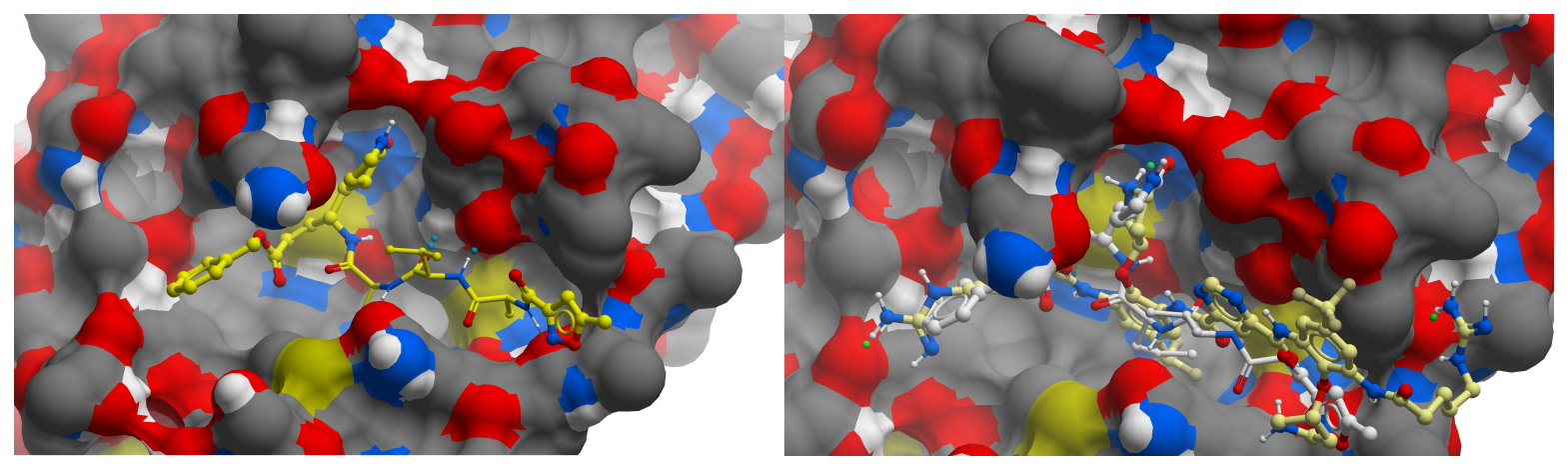

Figure 1: Ritonavir (left panel) and Brilacidin (right panel) docked within the binding site of SARS-CoV-2 Mpro. The receptor surface is colored as: red, oxygens; blue, nitrogen; white, polar hydrogen; grey, non-polar atoms. On the right panel, native ligand N3 (6LU7) is also displayed with white carbon atoms superimposed to Brilacidin (yellow carbons). Figure prepared with ICM (Molsoft LLC, San Diego, CA).
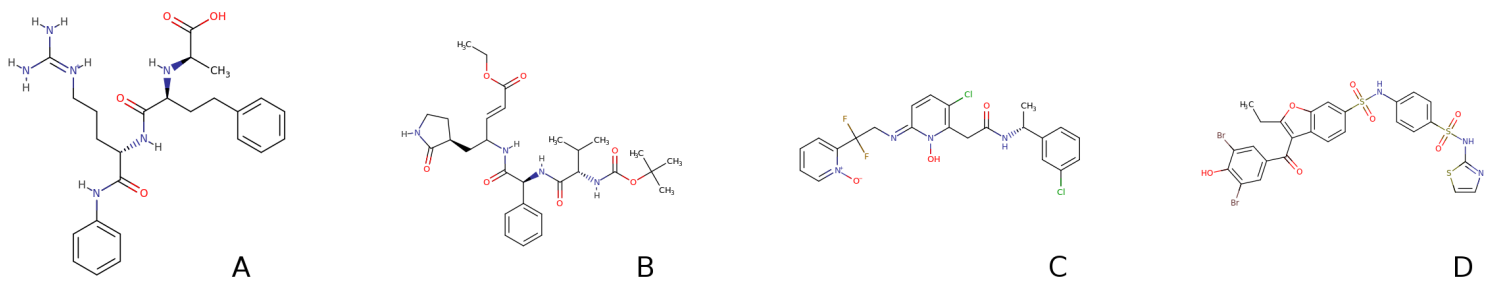

Figure 2: Experimental drugs identified as potential inhibitors of the SARS-CoV-2 Mpro: A, DB02747; B, DB04692; C, DB04722; D, DB03311

Since at the time of this writing there is no experimental structure available of SARSCoV-2 PL pro, this protease was modeled by homology based on the experimental crystal structure of SARS-CoV PL ${ }^{\text {pro }}$ complexed with the benzamide derivative GRL0617 ${ }^{24}$ (PDB 3E9S). Both proteases share $82 \%$ sequence identity, and $100 \%$ within the binding site. It should be highlighted that targeting $\mathrm{PL}^{\text {pro }}$ with antiviral drugs may not only block viral replication but also could inhibit the dysregulation of signaling cascades in infected cells 
Table 1: Potential inhibitors of SARS-CoV-2 Mpro from existing drugs and compounds undergoing clinical trials (DB, DrugBank; CH, ChEMBL).

\begin{tabular}{l|l|l}
\hline Drug name & Drug ID & Pharmacological function \\
\hline Felypressin & DB00093 & Vasoconstrictor \\
Angiotensinamide & DB13517 & Vasoconstrictor \\
Briladicin & CH2219413 & Head and neck neoplasms \\
Ritonavir & DB00503 & HIV-protease inhibitor \\
Samatasvir & CH3039519 & Hepatitis C infection \\
Indinavir & DB00224 & HIV-protease inhibitor \\
CR665 & DB05155 & $\kappa$-opioid receptor agonists \\
Lopinavir & CH729 & HIV-protease inhibitor \\
& DB02747 & N/A \\
& DB04692 & N/A \\
& DB04722 & N/A \\
& DB03311 & N/A \\
\hline
\end{tabular}

that may lead to cell death in other uninfected cells. ${ }^{25}$

From our analysis based on the criteria mentioned above, we identified several potential inhibitors of PL ${ }^{\text {pro }}$, of which we can highlight the following: Pilaralisib (CHEMBL3360203), an orally available selective small-molecule that inhibits the phosphoinositide-3 kinase (PI3K), that has been used in trials studying the treatment of different types of cancer, such as lymphoma, solid tumors, and glioblastoma; Tiracizine (DB13635), a dibenzazepine and an experimental anti-arrhythmic agent (Figure 3); PF-610355 (DB11871), an inhalable longacting $\beta_{2}$ adrenoreceptor agonist that was investigated some years ago for the treatment of asthma; Compound DB07665, an experimental drug which targets prothrombin; Cilazapril (DB01340), an FDA-appoved ACE inhibitor for the treatment of hypertension and heart failure (cilazapril is in fact a prodrug that is hydrolyzed to its main metabolite cilazaprilat; however, the structural differences of the prodrug and its active form share the same pose within the binding site); Indisulam (CHEMBL77517), a sulfonamide investigational compound with potential antineoplastic activity (Figure 3), which inhibits the cyclin-dependent kinase (CDK), which is usually over-expressed in cancerous cells; Compound DB03082, an experimental naphtamide derivative which shows activity as a urokinase-type plasminogen activator; Denibulin (DB05932), an investigational molecule under development for the treat- 


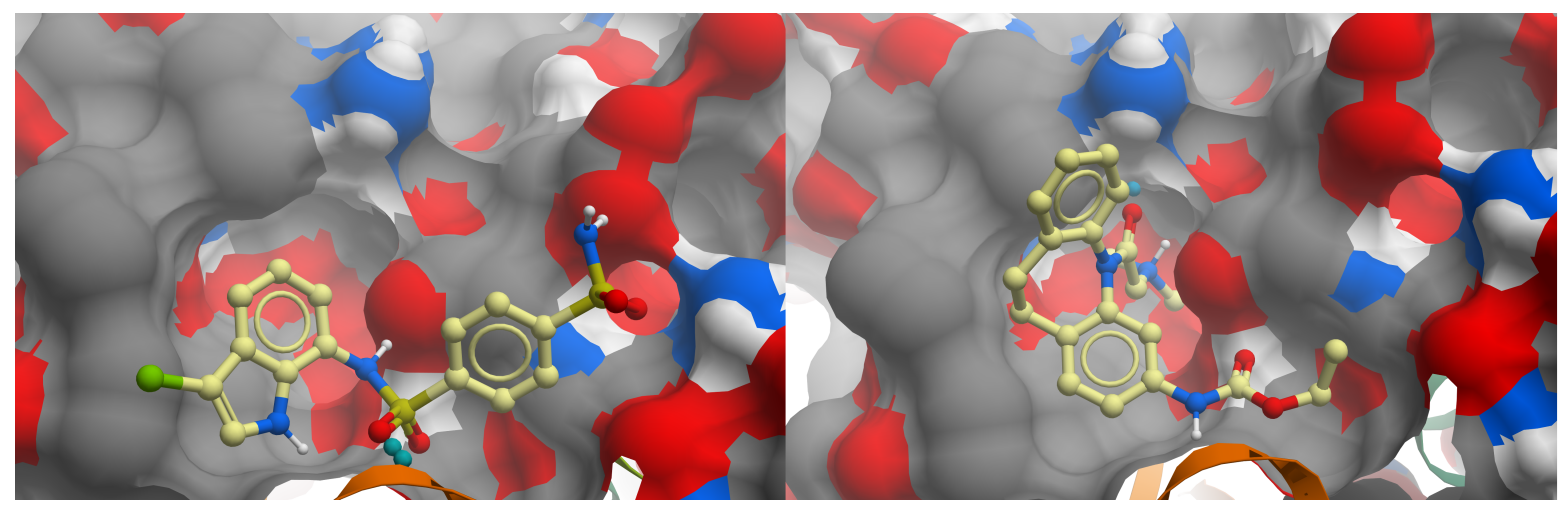

Figure 3: Indisulam (left panel) and Tiracizine (right panel) docked within the binding site of SARS-CoV-2 PL ${ }^{\text {pro }}$. The receptor surface is colored as: red, oxygens; blue, nitrogen; white, polar hydrogen; grey, non-polar atoms. The surface corresponding to amino-acids 270-273 (which forms the other wall of the channel where the molecules lie) is omitted for the sake of clarity. Figure prepared with ICM (Molsoft LLC, San Diego, CA).

ment of solid tumor cancers; Picotamide (DB13327), an experimental antiplatelet agent which acts as a thromboxane synthase inhibitor and a thromboxane receptor inhibitor. There are several other benzamide derivatives experimental drugs which can be considered as candidates, such as DB01748, DB08656, DB02861, CHEMBL1257015, and DB02429; moreover, benzamides have been reported to bind also to SARS-CoV and MERS-CoV PL pro. ${ }^{26}$

A list of potential inhibitors for SARS-CoV-2 of PL pro is summarized in Table 2.

The SARS-CoV-2 spike-protein (S-protein) receptor binding domain (RBD) binds to the

Table 2: Potential inhibitors of SARS-CoV-2 PL pro from existing drugs and compounds undergoing clinical trials (DB, DrugBank; CH, ChEMBL).

\begin{tabular}{l|l|l}
\hline Drug name & Drug ID & Pharmacological function \\
\hline Pilaralisib & CH3360203 & PI3K inhibition \\
Tiracizine & DB13635 & Anti-arrhythmic agent \\
PF-610355 & DB11871 & $\beta_{2}$ adrenoreceptor agonist \\
Cilazapril & DB01340 & ACE inhibitor for hypertension \\
Indisulam & CH77517 & Antineoplastic activity \\
Denibulin & DB05932 & Treatment of solid tumor cancers \\
Picotamide & DB13327 & Thromboxane synthase inhibitor \\
& DB01748 & N/A \\
& DB08656 & N/A \\
& DB02861 & N/A \\
& DB02429 & N/A \\
\hline
\end{tabular}


ACE2 receptor in the host cell, and thus may represent an attractive drug target. Experimental structures of the RBD in complex with ACE2 have been very recently obtained, ${ }^{27,28}$ so we isolated the RBD from one of them (PDB 6LZG), and docked and scored our library of approved drugs. It should be borne in mind that the RBD interface that should be targeted to preclude its interaction with ACE2 is not a buried binding site, a somehow shallow surface. Thus, the top scoring compounds are usually large and flexible molecules (such as Somastostatin and Daptomycin), and further validation using, for example, molecular dynamics, should be considered for prioritizing compounds before experimental evaluation. Potential inhibitors include Pralatrexate (DB06813), which is an approved drug for the treatment of relapsed or refractory peripheral T-cell lymphoma, Carumonam (DB13553), an experimental antimicrobial drug, Bradykinin (DB12126), which has been investigated for the treatment of hypertension and type 2 diabetes, Aclerastide (DB12631), an investigational drug for the treatment of diabetic foot, angiotensin II (DB11842), and granotapide (CHEMBL 4297618), which has been used in trials for the treatment of type 2 diabetes mellitus.

\section{Conclusions}

The infectous respiratory disease COVID-19 originated in Wuhan, China in December 2019, and rapidly expanding throughout the world is a serious threat to global health, totalizing at the time of this writing more than 1.5 million infected people, and more than 80,000 fatalities. So far, neither a vaccine nor an approved drug are currently available to treat this disease. Considering the time required to develop any of those options, drug repurposing seems the most appealing and straightforward approach. In this contribution, we performed dockingbased virtual screening on three SARS-CoV-2 targets (the proteases $\mathrm{M}^{\text {pro }}$ and PL ${ }^{\text {pro }}$, and the spike glycoprotein) using an in-house library of approved FDA drugs and investigational and experimental drugs to suggest potential compounds which may act as antivirals. While further experimental validation and clinical trials are necessary to confirm their activity 
against COVID-19, we join our effort to that of many other researchers to come up with an urgent and effective solution to this threat.

\section{Methods}

\section{Molecular system setup}

The structures of the SARS-CoV-2 M ${ }^{\text {pro }}$ bound to peptide inhibitor N3 (PDB 6LU7) and unbound (PDB 6YB7), the SARS-CoV-2 S-protein bound to ACE2 (PDB 6M17), and the SARS-CoV (PL ${ }^{\text {pro }}$ ) complexed with ligand GRL0617 (PDB 3E9S) were downloaded from the Protein Data Bank (PDB) and prepared using the ICM software ${ }^{29}$ (MolSoft, San Diego, CA, 2019) in a similar fashion as in earlier works. ${ }^{30}$ Succinctly, hydrogen atoms were added, followed by a short local energy minimization in the torsional space; the positions of polar and water hydrogens were determined by optimizing the hydrogen bonding network, and then all water molecules were deleted. All Asp and Glu residues were assigned a -1 charge, and all Arg and Lys residues were assigned a +1 charge. Histidine tautomers were chosen according to their corresponding hydrogen bonding pattern.

\section{Database preparation}

The docking library was constructed by merging molecule subsets from four different libraries: i) $\mathrm{ChEMBL}^{31}$ (Version 26) (including molecules which reached at least phases 1 , 2, 3 or 4); ii) DrugBank Version 5.1.5. ${ }^{32}$ (including chemical databases of Approved, Experimental and Investigational drugs); iii) the DrugCentral database of approved drugs; ${ }^{33}$ iv) the FDA-approved library from Selleck Chem. ${ }^{34}$ Redundant entries were eliminated, and metals and molecules with less than six and more than 120 atoms were deleted, totalizing 11552 molecules. 


\section{Homology modeling}

The SARS-CoV-2 PL pro was modeled using the SARS-CoV PL pro bound to benzamide derivative GRL0617 (3E9S) as template. The two proteins are highly similar (82\% sequence identity), with a $100 \%$ conservation within the binding site (Figure 4). A crude model was built, and then refined through local energy minimization using ICM. Taking into account the binding site conservation, and to avoid pocket collapse, ligand GRL0617 was kept during the refinement process, in a ligand-steered modeling fashion. ${ }^{30,35,36}$

\section{Molecular docking and quantum mechanical scoring}

Protein-molecule complexes were generated through flexible-ligand:rigid-receptor molecular docking with ICM, followed by structural relaxation to optimize protein-molecule interactions through cycles of local energy minimization; in this local energy minimization, amino-acids within $4 \AA$ of any docked molecule were considered free. For each target, all amino-acids within $8 \AA$ of any docked molecule were then collected, and used to build a reduced system, capping the N- and C-terminal of each fragment of the cut-out system with hydrogens (cf. Ref. ${ }^{7}$ for details on the relaxation process and system cutout).

The quantum mechanical docking score ${ }^{7}(Q M D S)$ on the reduced system was calculated according to

$$
Q M D S=\Delta G_{o}^{Q M}+\Delta G_{\text {conf }}^{Q M}(P)+\Delta G_{\text {conf }}^{Q M}(L)-T \Delta S,
$$

where the "o" subscript in the first term refers to the difference of the free energy calculated using the protein-ligand $(\mathrm{PL})$, protein $(\mathrm{P})$ and ligand $(\mathrm{L})$ conformations from the docked complex, the fourth term corresponds to the change in conformational entropy, and the second and third terms are calculated as

$$
\Delta G_{c o n f}^{Q M}(X)=G_{o}^{Q M}(X)-G^{Q M}(X),(\mathrm{X}=\mathrm{L}, \mathrm{P})
$$

where $G_{o}^{Q M}(X)$ is the energy of the isolated X in the conformation of the docked PL complex, 
PLpro_CoV

PLpro_CoV_2 PLpro_CoV

$\begin{array}{ll} & \\ \text { PLpro_CoV } & 59 \\ \text { PLpro_CoV_2 } & 61 \\ \text { PLpro_CoV } & \end{array}$

Lpro_CoV PLpro_CoV_2 PLPro_CoV

PLpro_CoV PLpro_CoV_2 PLpro_CoV

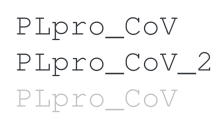

...EV+TIKVFTTVDN . NLHTQ\#VDMSMTYGQQFGP TYLDGADVTKIKPH . HEGKTF\#V ASMEVKTIKVFTTVDNTNLHTQLVDMSMTYGQQFGP TYLDGADVTKIKP HVNHEGKTFFV SLREVRTIKVFTTVDNINLHTQVVDMSMTYGQQFGP TYLDGADVTKIKP HNS HEGKTFYV

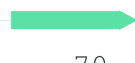

80

90

100

110

LP.DDTLR. EAFEYYHT.D. SF LGRYMSALNHTKKWK\#PQV . GLTSIKWADNN . YL . .\#L 59 LPSDDTLRSEAFEYYHTLDESF LGRYMSALNHTKKWKFP QVGGLTS IKWADNNXYLSSVL LPNDDTLRVEAFEYYHTTDP SF LGRYMSALNHTKKWKYP QVNGLTS IKWADNNCYLATAL 130 140 150 160 170

1

L. LQQ\#E\#KFN\#PALQ-AYYRARAG-AANFCALILAY . NKTVGELGDVRETM. \#L\#QHAN 119 LALQQLEVKFNAPALQEAYYRARAGDAANFCALILAYSNKTVGELGDVRETMTHLLQHAN 121 LTLQQIELKFNPPALQDAYYRARAGEAANFCALILAYCNKTVGELGDVRETMSYLFQHAN

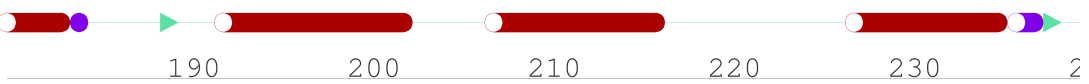

L-S\#KRVLNVVCK . CGQ . . TTL. GVEAVMYMGTLSY- .\#K. GV. IPC. CG+ . AT . YLVQQ 179 LESAKRVLNVVCKHCGQKTTTLTGVEAVMYMGTLSYDNLKTGVS IPCVCGRDATQYLVQQ 181 LDSCKRVLNVVCKTCGQQQTTLKGVEAVMYMGTLSYEQFKKGVQIPCTCGKQATKYLVQQ

$250 \quad 260 \quad 270 \quad 280 \quad 290$

ES.FVMMSAPPA.Y.L..GTF.CA.EYTGNYQCGHY.HIT.KETLY.IDGA\#LTK.SEYK 239 ESSFVMMSAPPAEYKLQQGTFLCANEYTGNYQCGHYTHITAKETLYRIDGAHLTKMSEYK 241 ESPFVMMSAPPAQYELKHGTFTCASEYTGNYQCGHYKHITSKETLYCIDGALLTKSSEYK

$>$ 310

GP\#TDVFYKE.SYTTTI

299 GPVTDVFYKETSYTTTI

301 GPITDVFYKENSYTTTI

$\Longrightarrow-\mathrm{D}$

Figure 4: Sequence alignment of the papain-like proteases $\mathrm{PL}^{\text {pro }}$ corresponding to SARS-CoV and SARS-CoV-2. Amino-acid conservation is shown on top (the sequence identity is $82 \%$ ). It can be observed that amino-acids in the binding site of SARS-CoV in contact with ligand GRL0617 (shown in grey) are fully conserved in SARS-CoV-2. A schematic representation of the secondary structure, corresponding to the template structure of SARS-CoV PL ${ }^{\text {pro }}$ (PDB 3E9S), is shown below the secuences as cylinders (helices) and arrows ( $\beta$-sheets). Figure prepared with ICM (Molsoft LLC, San Diego, CA)

and $G^{Q M}(X)$ is the energy of $\mathrm{X}$ in the free unbound state. The unbound states were generated through local energy minimization on both protein and molecule in isolation. The binding conformational entropy was calculated as

$$
\Delta S=-R \ln \Omega
$$


assuming that upon binding the molecule adopts a single conformational state (thus $S_{\text {bound }}=$ 0 ), and where $\Omega$ is the number of diverse torsional conformations of the molecule in its free state, calculated by performing a Monte-Carlo (MC) sampling with local energy minimization in the torsional space using ICM, ${ }^{29,37}$ and collecting all structurally different conformations within the lowest $3 \mathrm{kcal} / \mathrm{mol}$ energy (it is assumed that all conformers are equally probable).

QM calculations were performed using the MOPAC2016 software ${ }^{38}$ and its linear-scaling module MOZYME ${ }^{39}$ using the semi-empirical PM7 Hamiltonian. ${ }^{40}$ The solvation energy contribution in aqueous environment was calculated according to the COnductor Like Screening Model (COSMO) ${ }^{41}$ continuum solvent model, with default atomic radii and surface tension parameters. The solvent-accessible surface area was extracted from the MOPAC2016 output (cf. http://openMOPAC.net for details).

\section{Acknowledgement}

This work was supported by the National Agency for the Promotion of Science and Technology (ANPCyT) (PICT-2014-3599 and PICT-2017-3767). CNC thanks Molsoft LLC (San Diego, CA) for providing an academic license for the ICM program. The authors are grateful to the National System of High Performance Computing (Sistemas Nacionales de Computación de Alto Rendimiento, SNCAD), and the Centro de Cálculo de Alto Desempeño (Universidad Nacional de Córdoba) for granting use of their computational resources.

\section{References}

(1) Paules, C. I.; Marston, H. D.; Fauci, A. S. Coronavirus Infections-More Than Just the Common Cold. JAMA 2020, 323, 707-708.

(2) World Health Organisation, Novel Coronavirus (2019-nCoV) situation reports. https://www.who.int/emergencies/diseases/ 
novel-coronavirus-2019/situation-reports/.

(3) Lim, W. S. In ERS Handbook of Respiratory Medicine; Palange, P., Rohde, G., Eds.; European Respiratory Society, 2019; pp 393-399.

(4) Liu, Y.; Gayle, A. A.; Wilder-Smith, A.; Rocklöv, J. The reproductive number of COVID-19 is higher compared to SARS coronavirus. Journal of Travel Medicine 2020, 27, taaa021.

(5) Li, G.; De Clercq, E. Therapeutic options for the 2019 novel coronavirus (2019-nCoV). Nat Rev Drug Discov 2020, 19, 149-150.

(6) Wang, M.; Cao, R.; Zhang, L.; Yang, X.; Liu, J.; Xu, M.; Shi, Z.; Hu, Z.; Zhong, W.; Xiao, G. Remdesivir and chloroquine effectively inhibit the recently emerged novel coronavirus (2019-nCoV) in vitro. Cell Res. 2020, 30, 269-271.

(7) Cavasotto, C. N.; Aucar, M. G. High-throughput docking using quantum mechanical scoring. Frontiers in Chemistry 2020, 8, 246.

(8) Cavasotto, C. N.; Adler, N. S.; Aucar, M. G. Quantum Chemical Approaches in Structure-Based Virtual Screening and Lead Optimization. Frontiers in Chemistry 2018, 6, 188.

(9) Aucar, M. G.; Cavasotto, C. Molecular Docking Using Quantum Mechanical-Based Methods. Methods in Molecular Biology 2020, 2114, 269-284.

(10) Wang, J. ChemRxiv, 2020, DOI: 10.26434/chemrxiv.11875446.v1.

(11) Contini, A. ChemRxiv, 2020, DOI: 10.26434/chemrxiv.11847381.v1.

(12) Fischer, A.; Sellner, M.; Neranjan, S.; Lill, M. A.; Smieško, M. ChemRxiv, 2020, DOI: 10.26434/chemrxiv.11923239.v1.

(13) Smith, M.; Smith, J. C. ChemRxiv, 2020, DOI: 10.26434/chemrxiv.11871402.v4. 
(14) Wu, C.; Liu, Y.; Yang, Y.; Zhang, P.; Zhong, W.; Wang, Y.; Wang, Q.; Xu, Y.; Li, M.; Li, X.; Zheng, M.; Chen, L.; Li, H. Acta Pharmaceutica Sinica B, 2020, DOI: https://doi.org/10.1016/j.apsb.2020.02.008.

(15) Zhou, Y.; Hou, Y.; Shen, J.; Huang, Y.; Martin, W.; Cheng, F. Network-based drug repurposing for novel coronavirus 2019-nCoV/SARS-CoV-2. Cell Discovery 2020, 6, 14.

(16) Xu, Z.; Peng, C.; Shi, Y.; Zhu, Z.; Mu, K.; Wang, X.; Zhu, W. bioRxiv, 2020, DOI: $10.1101 / 2020.01 .27 .921627$.

(17) Khaerunnisa, S.; Kurniawan, H.; Awaluddin, R.; Suhartati, S.; Soetjipto, S. Preprints, 2020, DOI: $10.20944 /$ preprints202003.0226.v1.

(18) Jin, Z. et al. bioRxiv, 2020, DOI: 10.1101/2020.02.26.964882.

(19) Cavasotto, C. N.; Singh, N. Docking and High Throughput Docking: Successes and the Challenge of Protein Flexibility. Curr. Comput.-Aided Drug Design 2008, 4, 221-234.

(20) Spyrakis, F.; Cavasotto, C. N. Open challenges in structure-based virtual screening: Receptor modeling, target flexibility consideration and active site water molecules description. Archives of Biochemistry and Biophysics 2015, 583, 105-119.

(21) Cavasotto, C. N.; Abagyan, R. A. Protein Flexibility in Ligand Docking and Virtual Screening to Protein Kinases. Journal of Molecular Biology 2004, 337, 209-225.

(22) Cavasotto, C. N.; Kovacs, J. A.; Abagyan, R. A. Representing receptor flexibility in ligand docking through relevant normal modes. Journal of the American Chemical Society 2005, 127, 9632-9640.

(23) http://www.ipharminc.com/press-release/.

(24) Mesecar, A.; Ratia, K.; Pegan, S. A new class of papain-like protease/deubiquitinase inhibitors blocks SARS virus replication. to be published 2020, 
(25) Baez-Santos, Y. M.; St.John, S. E.; Mesecar, A. D. The SARS-coronavirus papain-like protease: Structure, function and inhibition by designed antiviral compounds. Antiviral Research 2015, 115, $21-38$.

(26) Lee, H.; Lei, H.; Santarsiero, B. D.; Gatuz, J. L.; Cao, S.; Rice, A. J.; Patel, K.; Szypulinski, M. Z.; Ojeda, I.; Ghosh, A. K.; Johnson, M. E. Inhibitor Recognition Specificity of MERS-CoV Papain-like Protease May Differ from That of SARS-CoV. ACS Chemical Biology 2015, 10, 1456-1465, PMID: 25746232.

(27) Yan, R.; Zhang, Y.; Li, Y.; Xia, L.; Guo, Y.; Zhou, Q. Structural basis for the recognition of SARS-CoV-2 by full-length human ACE2. Science 2020, 367, 1444-1448.

(28) Wang, Q.; Song, H.; Qi, J. Structure of novel coronavirus spike receptor-binding domain complexed with its receptor ACE2. to be published 2020,

(29) Abagyan, R.; Totrov, M. Biased probability Monte Carlo conformational searches and electrostatic calculations for peptides and proteins. J. Mol. Biol. 1994, 235, 983-1002.

(30) Phatak, S. S.; Gatica, E. A.; Cavasotto, C. N. Ligand-steered modeling and docking: A benchmarking study in Class A G-Protein-Coupled Receptors. Journal of Chemical Information and Modeling 2010, 50, 2119-2128.

(31) Gaulton, A. et al. The ChEMBL database in 2017. Nucleic Acids Research 2017, 45, D945-D954.

(32) Wishart, D. S. et al. DrugBank 5.0: a major update to the DrugBank database for 2018. Nucleic Acids Research 2018, 46, D1074-D1082.

(33) Ursu, O.; Holmes, J.; Knockel, J.; Bologa, C. G.; Yang, J. J.; Mathias, S. L.; Nelson, S. J.; Oprea, T. I. DrugCentral: online drug compendium. Nucleic Acids Research 2017, 45, D932-D939.

(34) https://www.selleckchem.com/screening/fda-approved-drug-library.html. 
(35) Cavasotto, C. N.; Orry, A. J. W.; Murgolo, N. J.; Czarniecki, M. F.; Kocsi, S. A.; Hawes, B. E.; O’Neill, K. A.; Hine, H.; Burton, M. S.; Voigt, J. H.; Abagyan, R. A.; Bayne, M. L.; Monsma, F. J. Discovery of Novel Chemotypes to a G-Protein-Coupled Receptor through Ligand-Steered Homology Modeling and Structure-Based Virtual Screening. Journal of Medicinal Chemistry 2008, 51, 581-588, PMID: 18198821.

(36) Cavasotto, C. N.; Palomba, D. Expanding the horizons of G protein-coupled receptor structure-based ligand discovery and optimization using homology models. Chem. Commun. 2015, 51, 13576-13594.

(37) Abagyan, R.; Totrov, M.; Kuznetsov, D. ICM - a New Method For Protein Modeling and Design - Applications to Docking and Structure Prediction From the Distorted Native Conformation. J. Comput. Chem. 1994, 15, 488-506.

(38) Stewart, J. J. P. MOPAC2016. 2016; http://OpenMOPAC.net.

(39) Stewart, J. J. P. Application of Localized Molecular Orbitals to the Solution of Semiempirical Self-Consistent Field Equations. Int. J. Quant. Chem. 1996, 58, 133-146.

(40) Stewart, J. J. P. Optimization of parameters for semiempirical methods VI: more modifications to the NDDO approximations and re-optimization of parameters. J. Mol. Model. 2013, 19, 1-32.

(41) Klamt, A.; Schüürmann, G. COSMO: A New Approach to Dielectric Screening in Solvents with Explicit Expressions for the Screening Energy and its Gradient. J. Chem. Soc. Perkin Trans. 1993, 2, 799-805. 